City University of New York (CUNY) CUNY Academic Works

1982

\title{
Nonperturbative proof of the non-Abelian anomalies
}

\author{
A. P. Balachandran \\ Syracuse University \\ G. Marmo \\ Syracuse University \\ V. P. Nair \\ CUNY City College \\ C. G. Trahern \\ Syracuse University
}

\section{How does access to this work benefit you? Let us know!}

More information about this work at: https://academicworks.cuny.edu/cc_pubs/378

Discover additional works at: https://academicworks.cuny.edu

This work is made publicly available by the City University of New York (CUNY).

Contact: AcademicWorks@cuny.edu 
Nonperturbative proof of the non-Abelian anomalies

\author{
A. P. Balachandran, G. Marmo,* V. P. Nair, and C. G. Trahern \\ Physics Department, Syracuse University, Syracuse, New York 13210 \\ (Received 16 September 1981)
}

We give a nonperturbative derivation of non-Abelian anomalies.

\section{INTRODUCTION}

The non-Abelian axial anomalies ${ }^{1,2}$ have played an increasingly important role in the study of gauge theories. A nonperturbative derivation of these anomalies however has not appeared in the published literature. In this note, we try to supply such a derivation. (Similar results have been obtained by S. Coleman and B. Grossman ${ }^{3}$ using different techniques.)

Our approach to the problem relies on some recent work of Vergeles and Fujikawa. ${ }^{4}$ They have pointed out that the anomaly in any axial-vector current is a result of the noninvariance of the fermion functional measure in the path integral under the corresponding axial transformation. It can in fact be expressed in terms of the associated Jacobian determinant. Here, we shall evaluate this determinant using $\zeta$-function regularization. ${ }^{5}$ The final results agree with those of Bardeen. ${ }^{1}$

In Sec. II we recall the work of Ref. 4 and write down the formal expression for the anomalies. The Wess-Zumino consistency conditions ${ }^{6}$ are derived from the properties of Jacobians. In Sec. III the continuation of the chiral group $\mathrm{U}_{L}(N) \times \mathrm{U}_{R}(N)$ to Euclidean space is discussed. We find that it must be continued to $\operatorname{GL}(N, C)$ to maintain the Hermiticity of the Dirac operator. In Sec. IV we outline the evaluation of the Jacobian. Section $\mathrm{V}$ concludes the paper with some brief comments.

\section{THE ANOMALY AS A JACOBIAN}

We consider the gauge theory of $N$ massless Dirac fields $\psi^{a}(a=1,2, \ldots, N)$ where the gauged group $G$ is the chiral group $\mathrm{U}_{L}(N) \times \mathrm{U}_{R}(N)$. The field $\psi=\left(\psi^{1}, \psi^{2}, \ldots, \psi^{N}\right)$ transforms according to the representation $(N, 1)+(1, N)$ of this group. A basis for the Lie algebra $\underline{G}$ of $G$ in this representation is $i \lambda^{a}, i \gamma_{5} \lambda^{a}$ where $\lambda^{a}$ are the Gell-Mann matrices. The vector and axial-vector gauge fields are

$$
V_{\mu}=-i V_{\mu}^{a} \lambda^{a}, A_{\mu}=-i A_{\mu}^{a} \lambda^{a}
$$

so that the covariant derivative of $\psi$ is

$$
D_{\mu} \psi \equiv\left(\partial_{\mu}+V_{\mu}+\gamma_{5} A_{\mu}\right) \psi \text {. }
$$

According to Ref. 4, the fermion functional measure $d \psi d \bar{\psi}$ is not in general invariant under the gauge transformations associated with $G$. Thus if

$$
\psi^{ \pm}=\exp \left(\frac{1 \pm \gamma_{5}}{2} \lambda^{a} \theta_{a}\right) \psi, \quad \theta_{a}=\theta_{a}(x)
$$

then ( $T r$ will denote trace over Dirac and internal indices as well as over $x$, while tr will denote trace over Dirac and/or internal indices only)

$$
\begin{aligned}
d \psi^{ \pm} d \bar{\psi} \pm & =d \psi d \bar{\psi} \operatorname{Det}\left[e^{\mp i \gamma_{5} \lambda^{a} \theta_{a}}\right] \\
& =d \psi d \bar{\psi} e^{\mp i \operatorname{Tr} \lambda^{a} \theta_{a} \gamma_{5}} .
\end{aligned}
$$

This anomalous transformation law is the cause of the anomalous nonconservation laws

$$
\begin{aligned}
& \int d^{4} x \theta_{a}(x) \partial^{\mu} l_{\mu}^{a}=\operatorname{Tr} \lambda^{a} \theta_{a} \gamma_{5}, \\
& \int d^{4} x \theta_{a}(x) \partial^{\mu} r_{\mu}^{a}=-\operatorname{Tr} \lambda^{a} \theta_{a} \gamma_{5}
\end{aligned}
$$

of the currents $l_{\mu}^{a}$ and $r_{\mu}^{a}$ for the left-handed and right-handed transformations.

As a prelude to the derivation of the WessZumino consistency conditions, we note that the trace in (2.4) depends on the potentials. Thus $d \psi d \bar{\psi}$ also depends on the potentials. We exhibit this fact by writing

$$
\begin{aligned}
& d \psi d \bar{\psi}=d \mu(\psi, W), \\
& W_{\mu}=V_{\mu}+\gamma_{5} A_{\mu} .
\end{aligned}
$$

Let us also denote the gauge transform of $W$ by an element $g$ of the gauge group as $g \circ W$. For instance, if

$$
\begin{aligned}
& g=e^{\eta}, \\
& \eta=i \frac{1+\gamma_{5}}{2} \lambda^{a} \theta_{a}+i \frac{1-\gamma_{5}}{2} \lambda^{a} \phi_{a},
\end{aligned}
$$


then

$$
(g \circ W)_{\mu} \equiv g W_{\mu} g^{-1}+g \partial_{\mu} g^{-1} .
$$

The transformation law for the functional measure can now be expressed as

$$
d \mu(g \psi, g \circ W)=d \mu(\psi, W) e^{\beta(g, W)},
$$

where for (2.7),

$$
\beta(g, W)=-i \operatorname{Tr} \lambda^{a}\left(\theta_{a}-\phi_{a}\right) \gamma_{5} .
$$

Since

$d \mu\left(\left(g g^{\prime}\right) \psi,\left(g \circ g^{\prime}\right) \circ W\right)=d \mu\left(g\left(g^{\prime} \psi\right), g \circ\left(g^{\prime} \circ W\right)\right)$ we find

$$
\beta\left(g g^{\prime}, W\right)=\beta\left(g, g^{\prime} \circ W\right)+\beta\left(g^{\prime}, W\right)
$$

(modulo $2 \pi$ ).

Equation (2.12) is an integrated form of the Wess-Zumino conditions. To derive the latter, we start from the identity

$$
\begin{aligned}
\beta\left(g g^{\prime} g^{-1}, W\right)= & \beta\left(g, g^{\prime} \circ g^{-1} \circ W\right) \\
& +\beta\left(g^{\prime}, g^{-1} \circ W\right)+\beta\left(g^{-1}, W\right)
\end{aligned}
$$

which follows from (2.12). Set $g=e^{\eta}, g^{\prime}=e^{\epsilon}$ and consider $\eta$ and $\epsilon$ to be small. Then up to leading terms,

$$
\beta(1+[\eta, \epsilon]+\cdots, W)=\delta_{\eta} \beta(1+\epsilon+\cdots, W)-\delta_{\epsilon} \beta(1+\eta+\cdots, W),
$$

where $\delta_{\epsilon} \beta$ is the change in $\beta$ due to an infinitesimal gauge transformation with parameter $\epsilon$ :

$$
\delta_{\epsilon} \beta(s, W)=\text { term linear in } \epsilon \text { in } \beta(s, W-[\epsilon, W]-\partial \epsilon)-\beta(s, W) .
$$

Now, for any group element $s$ of the form $1+\xi+O\left(\xi^{2}\right), \beta(s, W)$ and $\beta\left(e^{\xi}, W\right)$ are the same up to the linear term in $\xi$ and are given by (2.10) and (2.7). Thus (2.14) is just the Wess-Zumino condition.

\section{THE DIRAC OPERATOR AND CHIRAL GROUP IN EUCLIDEAN SPACE}

The regularization of the trace in (2.4) will involve us in the consideration of the Euclideanspace Dirac operator

$$
\gamma_{\lambda} D_{\lambda}=\gamma_{\lambda}\left(\partial_{\lambda}+V_{\lambda}+\gamma_{5} A_{\lambda}\right)
$$

in the Hilbert space of functions with scalar product

$$
\left(\psi_{1}, \psi_{2}\right)=\int d^{4} x \psi_{1}^{\dagger}(x) \psi_{2}(x) .
$$

Our manipulations with the heat kernel will require this operator to be anti-Hermitian. The anti-Hermiticity of $\gamma \cdot D$ implies the properties

$$
V_{\lambda}^{\dagger}=-V_{\lambda}, \quad A_{\lambda}^{\dagger}=A_{\lambda}
$$

of $V_{\lambda}$ and $A_{\lambda}$ in Euclidean space. Thus the Hermiticity property of $A_{\lambda}$ has to be changed in the passage from Minkowski to Euclidean space.

If this Hermiticity property is to be preserved by gauge transformations in Euclidean space, then in (2.7) we have to regard $\theta_{a}+\phi_{a}$ as real and $\theta_{a}-\phi_{a}$ as pure imaginary when we work in Euclidean space. This changes the group from $\mathrm{U}_{L}(N) \times \mathrm{U}_{R}(N)$ to $\mathrm{GL}(N, C)$. This change is similar to the change that affects the group acting on the spinor index of a Dirac field in going from Minkowski to Euclidean space: the group is $\mathrm{SL}(2, C)$ in Minkowski and $\mathbf{S U}(2) \times \mathbf{S U}(2)$ in $\mathrm{Eu}-$ clidean space.

If the gauged group $G$ in Minkowski space is an arbitrary subgroup of $\mathrm{U}_{L}(N) \times \mathrm{U}_{R}(N)$, then we do not know how to continue it to Euclidean space. For instance if $G=U_{L}(N)$, then in (2.7), $\phi_{a}=0$. The natural Euclidean continuation of $\eta$ (with $\left.\phi_{a}=0\right)$ is $i\left[\frac{1}{2}\left(1+i \gamma_{5}\right)\right] \lambda^{a} \theta_{a}\left(\theta_{a}\right.$ being real $)$. However $i\left[\frac{1}{2}\left(1+i \gamma_{5}\right)\right] \lambda^{a}$ do not span a Lie algebra.

\section{EVALUATION OF THE ANOMALY}

We follow standard methods for the evaluation of the anomaly $\operatorname{Tr} \lambda^{a} \theta_{a} \gamma_{5}{ }^{5}$ It is continued to Euclidean space (where $\theta_{a}^{*}=-\theta_{a}$ ) and evaluated by $\zeta$-function regularization. It is finally continued back to Minkowski space (where $\theta_{a}^{*}=\theta_{a}$ ).

We now quickly review the $\zeta$-function regularization method. In Euclidean space, let $\phi_{n}$ be the eigenfunctions of $\gamma \cdot D$ :

$$
\begin{aligned}
& \gamma \cdot D \phi_{n}=i \lambda_{n} \phi_{n}, \\
& \left(\phi_{n}, \phi_{m}\right)=\delta_{n m}, \\
& \sum_{n} \phi_{n}(x) \phi_{n}^{\dagger}\left(x^{\prime}\right)=\delta^{4}(x-y) 1 .
\end{aligned}
$$


Then (formally),

$$
\begin{aligned}
& \operatorname{Tr} \lambda^{a} \theta_{a} \gamma_{5}=\int d^{4} x \theta_{a}(x) \lim _{\substack{s \rightarrow 0 \\
x \rightarrow y}} \operatorname{tr} \lambda^{a} \gamma_{5} \xi(s, x, y), \\
& \zeta(s, x, y) \equiv \sum_{n} \frac{\phi_{n}(x) \phi_{n}^{\dagger}(y)}{\lambda_{n}{ }^{2 s}} .
\end{aligned}
$$

The trace on the right side (tr with a lower case $t$ ) is only over Dirac and internal indices.

The $\zeta$ function can be written in terms of the heat kernel

$$
\begin{aligned}
& h(t, x, y)=\left\langle x\left|e^{t(\gamma \cdot D)^{2}}\right| y\right\rangle: \\
& \zeta(s, x, y)=\frac{1}{\Gamma(s)} \int_{0}^{\infty} \mathrm{dt} t^{s-1} h(t, x, y) .
\end{aligned}
$$

The heat kernel has the asymptotic expansion ${ }^{5}$

$$
\begin{aligned}
h(t, x, y)= & \frac{1}{16 \pi^{2} t^{2}} \exp \left[-\frac{(x-y)^{2}}{4 t}\right] \\
& \times \sum_{n=0}^{\infty} a_{n}(x, y) t^{n}
\end{aligned}
$$

for small $t$. Inserting (4.4) in (4.3), we find that

$$
\zeta(0, x, x)=\frac{a_{2}(x, x)}{16 \pi^{2}}
$$

and that

$$
\begin{aligned}
& \operatorname{Tr} \lambda^{a} \theta_{a} \gamma_{5}=\frac{1}{16 \pi^{2}} \int d^{4} x \theta_{a}(x) \operatorname{tr} \lambda^{a} \gamma_{5} a_{2}(x), \\
& a_{2}(x) \equiv a_{2}(x, x) .
\end{aligned}
$$

The coefficients $a_{n}$ can be evaluated recursively using the heat equation

$$
\partial_{t} h(t, x, y)=(\gamma \cdot D)^{2} h(t, x, y)
$$

and the boundary condition

$$
h(0, x, y)=\delta^{4}(x-y) 1 .
$$

It is known ${ }^{5}$ that if there is a differential operator

$$
\mathscr{D}_{\mu}=\partial_{\mu}+P_{\mu}
$$

such that

$$
X=(\gamma \cdot D)^{2}-\mathscr{D}^{2}
$$

involves no differential operator, then

$$
a_{2}(x)=\frac{1}{2} X^{2}+\frac{1}{12} Y_{\mu \nu} Y_{\mu \nu}+\frac{1}{6} \mathscr{D}^{2} X,
$$

where

$$
\mathscr{D}_{\mu} X=\partial_{\mu} X+\left[P_{\mu}, X\right]
$$

and

$$
\boldsymbol{Y}_{\mu \nu}=\left[\mathscr{D}_{\mu}, \mathscr{D}_{\nu}\right] \text {. }
$$

Thus in our problem, we have to find a $\boldsymbol{P}_{\boldsymbol{\mu}}$ such that $X$ in (4.9) involves no differential operator and then evaluate the anomaly from (4.10) and (4.5). A suitable $P_{\mu}$ and the corresponding $X$ are

$$
\begin{aligned}
P_{\mu}=V_{\mu}+\gamma_{5} A_{\mu}-\gamma_{5} \gamma \cdot A & \gamma_{\mu}, \\
X=\frac{1}{4}\left[\gamma_{\mu}, \gamma_{\nu}\right] F_{\mu \nu}+\gamma_{5}\{ & \partial_{\mu}\left(\gamma \cdot A \gamma_{\mu}\right) \\
+ & {\left.\left[V_{\mu}, \gamma \cdot A\right] \gamma_{\mu}\right\}+2 A^{2}, }
\end{aligned}
$$

where

$$
\begin{aligned}
& F_{\mu \nu}=V_{\mu \nu}+\gamma_{5} A_{\mu \nu}, \\
& V_{\mu \nu}=\partial_{\mu} V_{v}-\partial_{v} V_{\mu}+\left[V_{\mu}, V_{v}\right]+\left[A_{\mu}, A_{v}\right], \\
& A_{\mu \nu}=\partial_{\mu} A_{v}-\partial_{v} A_{\mu}+\left[V_{\mu}, A_{v}\right]-\left[V_{v}, A_{\mu}\right] .
\end{aligned}
$$

The calculation of the anomaly is now straightforward though tedious. We find (here $\left.[A, B]_{ \pm}=A B \pm B A\right)$

$$
\begin{aligned}
& \frac{1}{16 \pi^{2}} \operatorname{tr} \lambda^{a} \gamma_{5} a_{2}(x)=\operatorname{tr} \lambda^{a}\left(G^{(1)}+G^{(2)}\right), \\
& G^{(1)}=-\frac{1}{8 \pi^{2}} \epsilon_{\mu \nu \alpha \beta}\left[\frac{1}{4} V_{\mu \nu} V_{\alpha \beta}+\frac{1}{12} A_{\mu \nu} A_{\alpha \beta}-\frac{2}{3}\left(A_{\mu} A_{\nu} V_{\alpha \beta}+A_{\mu} V_{\nu \alpha} A_{\beta}+V_{\mu \nu} A_{\alpha} A_{\beta}\right)+\frac{8}{3} A_{\mu} A_{v} A_{\alpha} A_{\beta}\right], \\
& G^{(2)}=-\frac{1}{16 \pi^{2}}\left\{\frac{4}{3}\left[\left(D_{\mu}^{V} A_{\nu}+D_{\nu}^{V} A_{\mu}\right), A_{\mu} A_{v}\right]_{+}-\frac{2}{3}\left[D^{V} \cdot A, A^{2}\right]_{+}+\frac{4}{3}\left[A_{\mu}, D_{\lambda}^{V} V_{\mu \lambda}\right]_{-}-\frac{1}{3}\left[A_{\mu \lambda}, V_{\mu \lambda}\right]_{-}\right. \\
& \left.\quad+\frac{2}{3} D_{\rho}^{V} D_{\rho}^{V}\left(D^{V} \cdot A\right)+4 A_{\lambda}\left(D^{V} \cdot A\right) A_{\lambda}\right\}, \\
& D_{\mu}^{V} f \equiv \partial_{\mu} f+\left[V_{\mu}, f\right]_{-} .
\end{aligned}
$$


The term involving $G^{(1)}$ is identical to Bardeen's expression. ${ }^{1}$ The terms involving $G^{(2)}$ on the other hand can be removed from the divergence equations (2.5) (and hence Ward-Takahashi identities) by following an idea discussed by Bardeen. ${ }^{1}$ Thus we add the following counterterm $\Delta \mathscr{L}$ to the Lagrangian density:

$$
\begin{aligned}
\Delta \mathscr{L}=-\frac{1}{8 \pi^{2}} \operatorname{tr} & \left\{\frac{2}{3}\left(D_{\mu}^{V} A_{\nu}\right)^{2}\right. \\
& -\left(D^{V} \cdot A^{2}\right)^{2}-\frac{2}{3}\left[A_{\mu}, A_{\nu}\right]_{-}{ }^{2} \\
& \left.+\frac{2}{3}\left(A_{\mu} A_{\nu} A_{\mu} A_{\nu}\right)+\frac{1}{2} V_{\mu \nu}{ }^{2}\right\} .
\end{aligned}
$$

Under an axial transformation $\psi \rightarrow e^{i \gamma_{5} \lambda^{a} \theta_{a}} \psi$, the first-order variation of

$$
\int d^{4} x \Delta \mathscr{L}
$$

is precisely

$$
2 \int d^{4} x \theta_{a}(x) \operatorname{tr} \lambda^{a} G^{(2)}(x) .
$$

Thus the infinitesimal variation of

$$
d \mu(\psi, W) e^{i \int d^{4} x \Delta \mathscr{L}}
$$

under an axial transformation does not involve $G^{(2)}$. In other words, the Ward-Takahashi identities are not affected by $G^{(2)}$ when the counterterm $\Delta \mathscr{L}$ is added to the Lagrangian density. $(\Delta \mathscr{L}$ is invariant under the vector gauge group. Therefore, it does not generate anomalies for the vector currents.)

\section{CONCLUDING REMARKS}

The expression in (4.14) for the anomaly fulfills the consistency conditions. This follows from the fact that the contribution from $G^{(1)}$ can be verified to do so by an explicit calculation, ${ }^{6}$ while the contribution from $G^{(2)}$ does so since it can be reproduced by the variation of the action (4.16) (cf. Ref. 6). We feel that there should be a proof of such results which uses only the formal properties of the $\zeta$-function regularization and the heat equation. We also feel that the potential $P_{\mu}$ in (4.12) should be capable of a simple interpretation. Our attempts at the resolution of these problems have not however been successful.

\section{ACKNOWLEDGMENTS}

We thank P. P. Divakaran, S. Rajeev, and J. Schechter for discussions. G. Marmo thanks Joshua Goldberg and Kameshwar Wali for their warm hospitality at Syracuse University where this work was done. The work of A.P.B., V.P.N., and C.G.T. was supported by the U.S. Department of Energy under Contract No. DE-AC02-76ER03533. The work of G.M. was supported by INFN, Italy.
"Permanent address: Istituto di Fisica Teorica, Università di Napoli, Mostra d'Oltremare Pad. 19, 80125 Napoli, Italy.

${ }^{1}$ W. A. Bardeen, Phys. Rev. 184, 1848 (1969).

${ }^{2}$ D. Gross and R. Jackiw, Phys. Rev. D 6 , 477 (1972);

H. Georgi and S. L. Glashow, ibid. 6, 429 (1972).

${ }^{3}$ S. Coleman and B. Grossman (unpublished).

${ }^{4}$ S. N. Vergeles, quoted in A. A. Migdal, Phys. Lett.
81B, 37 (1979); K. Fujikawa, Phys. Rev. Lett. 44, 1733 (1980); Phys. Rev. D 21, 2848 (1980).

${ }^{5}$ N. K. Nielsen, NORDITA Report No. 78/24, 1978 (unpublished); N. K. Nielsen, M. T. Grisaru, H. Römer, and P. van Nieuwenhuizen, Nucl. Phys. B140, 477 (1978) and references therein.

6J. Wess and B. Zumino, Phys. Lett. 37B, 95 (1971). 